\section{PSICOLOGÍA IBEROAMERICANA}

Psicología Iberoamericana

ISSN: 1405-0943

psicología.iberoamericana@uia.mx

Universidad Iberoamericana, Ciudad de México

México

Romero Palencia, Angélica; del Castillo Arreola, Arturo; Solano Solano, Gloria; Rivera

Aragón, Sofía; Cruz del Castillo, Cinthia

Predictores del deseo sexual en una muestra de mujeres mexicanas

Psicología Iberoamericana, vol. 22, núm. 2, julio-diciembre, 2014, pp. 36-45

Universidad lberoamericana, Ciudad de México

Distrito Federal, México

Disponible en: http://www.redalyc.org/articulo.oa?id=133938134005

Cómo citar el artículo

- Número completo

- Más información del artículo

- Página de la revista en redalyc.org

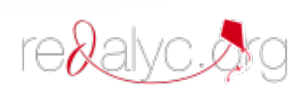

Sistema de Información Científica

Red de Revistas Científicas de América Latina, el Caribe, España y Portugal Proyecto académico sin fines de lucro, desarrollado bajo la iniciativa de acceso abierto 


\title{
Predictores del deseo sexual en una muestra de mujeres mexicanas
}

\author{
Predictors of Sexual Desire in a Sample of Mexican Women
}

\author{
Angélica Romero Palencia \\ Arturo del Castillo Arreola \\ Gloria Solano Solano \\ Universidad Autónoma del Estado de Hidalgo \\ Sofía Rivera Aragón \\ Universidad Nacional Autónoma de México \\ Cinthia Cruz del Castillo \\ Universidad Iberoamericana, Campus Santa Fe
}

\section{RESUMEN}

El deseo sexual es un energía psicobiológica (Levine, 2003) un interés en objetos o actividades sexuales (Regan \& Berscheid, 2000) y un constructo que existe y toma su forma debido a la cultura y la historia (Giles, 2006). Por tanto, el objetivo del presente estudio consistió en detectar si la satisfacción con la relación, los celos, la sociosexualidad y la conducta sexual son predictores confiables del deseo sexual en un grupo de mujeres heterosexuales mexicanas. Las variables incluidas en el modelo fueron los patrones de comportamiento sexual, satisfacción con la relación, celos e infidelidad. La muestra se conformó con 193 mujeres voluntarias, seleccionadas mediante un muestreo no probabilístico accidental, cuyas edades oscilan entre los 18 y los 64 años, con una relación de pareja heterosexual. Para probar los modelos se llevaron a cabo análisis de regresión múltiple. Los resultados mostraron la alta personalidad sexual conquistadora y la baja tendencia a la promiscuidad como los principales predictores del deseo sexual en las mujeres de la muestra.

Palabras clave: deseo sexual, mujeres, sexualidad, personalidad sexual.

\section{ABSTRACT}

Sexual desire is a psychobiological force (Levine, 2003), an interest in sexual activities or objects (Regan \& Berscheid, 2000) and a construct that exists and is shaped by culture and history (Giles, 2006). Therefore, this study aimed to detect whether satisfaction with a relationship, jealousy, sociosexuality and sexual behavior are reliable predictors of sexual desire in a group of heterosexual Mexican women. The variables included in the model were the patterns of sexual behavior, satisfaction with the relationship, jealousy and infidelity. The sample consisted of 193 women volunteers in a relationship with a heterosexual partner, aged between 18 and 64, who were selected by accidental non-probabilistic sampling. A multiple regression analysis was applied in order to test the models. The results showed that highly dominant sexual personalities and low tendency of promiscuity were the main predictors of sexual desire among women in the sample.

Keywords: Sexual Desire, Women, Sexuality, Sexual Personality. 


\section{INTRODUCCIÓN}

El deseo sexual o la motivación sexual se define formalmente como la energía psicobiológica que precede y acompaña la excitación y que tiende a crear la conducta sexual (Levine, 2003). Según Regan y Berscheid (2000), el deseo sexual es un interés en objetos o actividades sexuales como besar, masturbarse, tener coito o la excitación sexual como la lubricación vaginal. Mientras que Giles (2006), afirma que el deseo sexual es un término socialmente construido que existe y toma su forma debido a la cultura y a la historia.

De acuerdo con Levine (1992; 2003), el deseo sexual contribuye, entre otras cosas, a la evolución de la identidad sexual, es esencial para la formación de las relaciones sexuales, es una fuerza que ayuda a controlar/manejar muchas relaciones no sexuales, posibilita la procreación, es un barómetro de la vitalidad de la relación, nutre a los miembros de la pareja y está influido por circunstancias socialmente envolventes de la vida adulta que determinan en forma sutil si la gente piensa en su experiencia de deseo como un problema o no.

La falta de deseo sexual, por tanto, suele tener implicaciones en múltiples áreas de funcionamiento de los seres humanos. Estas implicaciones se pueden vivir de manera diferente en hombres y mujeres, dadas las variables fisiológicas, psicológicas y socioculturales involucradas.

En culturas tradicionales como la mexicana, la normatividad del deseo sexual sigue siendo distinta para hombres y mujeres. Todavía en la actualidad y a pesar de que las mujeres han ganado espacios en el área laboral y en espacios públicos (Rocha \& Cruz del Castillo, 2013), en el área privada se sigue castigando el ejercicio abierto de la sexualidad y se le vincula exclusivamente al ámbito de la reproducción, en el marco de una relación de pareja estable.

Hablar de deseo sexual, de erotismo y de conducta sexual no sólo se restringe cultural sino individualmente, por el desconocimiento de las mujeres de sus propios cuerpos, necesidades e intereses sexuales (Cruz del Castillo, Romero y Gil-Bernal, 2013), circunstancia que puede llevarlas a experimentar poco deseo sexual. Sin embargo, la falta de deseo sexual en las mujeres suele causarles angustia y puede tener efectos negativos en las relaciones personales, la calidad de vida y el estado de salud en general (Kingsberg, 2011).
Sánchez, Corres, Blum y Carreño (2009) compararon un grupo sin disfunciones sexuales y un grupo con deseo sexual hipoactivo. Los datos revelaron lo importante que es el deseo sexual tanto en el bienestar como en la falta de bienestar en las mujeres, ya que aquéllas con deseo sexual hipoactivo mostraron un decremento en la autoestima y un incremento en las características de sumisión, además de reportar problemas conyugales en la actualidad.

No obstante, este problema parece no ser exclusivo de la sociedad mexicana; hallazgos de grandes estudios que analizaron la prevalencia de los síntomas sexuales relacionados con la angustia en Estados Unidos y Europa, mostraron con claridad que la disfunción sexual femenina es una carga real para muchas mujeres y aproximadamente una de cada diez puede recibir un diagnóstico de trastorno de deseo sexual hipoactivo (тDsн) (Shifren, Monz, Russo, Segreti \& Johannes, 2008). Dennerstein, Hayes, Sand y Lehert (2009), realizaron otro estudio transversal con una muestra de mujeres estadounidenses y europeas entre 18 y 25 años de edad, en el que encontraron que las mujeres que padecían características del TDSH tenían patrones más negativos de interacciones de pareja.

Según datos recabados por Kamei y Kamei (2005), quienes aplicaron el Î́ndice de Función Sexual Femenina a 217 mujeres chilenas, se encontró que $67.7 \%$ presentó disfunción sexual femenina a través de la alteración de uno o más de los siguientes aspectos: sin deseo sexual 52.9\%; ausencia de orgasmo $37.3 \%$; falta de lubricación 28.6\%; dispareunia 17.9\%; falta de excitación $24.8 \%$; y falta de satisfacción sexual $78.8 \%$. Dentro de este mismo estudio se detectó que $80.6 \%$ consideró la relación sexual importante para sus relaciones de pareja.

Basson (2001) desarrolló un modelo no lineal de la respuesta sexual femenina que integra la intimidad emocional, los estímulos sexuales y la satisfacción con la relación, además tiene en cuenta las numerosas razones de las mujeres para iniciar o estar receptivas a un encuentro sexual. Este autor encontró que las mujeres pueden involucrarse en la actividad sexual para aumentar la cercanía emocional y el compromiso, por ejemplo, sin haber tenido un deseo previo o pensamientos acerca de actividades sexuales. Esta decisión conduce a enfocarse en el estímulo sexual que se pro- 
cesa en la mente y que se ve influido por diversos factores biológicos y psicológicos.

La experiencia femenina de deseo sexual es una cuestión multidimensional, originada e influida por múltiples factores. Las mujeres construirán su propia definición de lo que es el deseo sexual normal, con base en su cultura, antecedentes y experiencias sexuales, así como en su propio impulso biológico (Kingsberg, 2011), y la interacción con su pareja (Romero, Cruz del Castillo \& Díaz Loving, 2008). Por tanto, el objetivo del presente estudio consistió en detectar si la satisfacción con la relación, los celos, la sociosexualidad y la conducta sexual son predictores confiables del deseo sexual en un grupo de mujeres heterosexuales mexicanas.

\section{MÉTODO}

\section{Participantes}

Participaron 193 voluntarias seleccionadas mediante un muestreo no probabilístico accidental, cuyas edades oscilaban entre los 18 y los 64 años (con un promedio de 38 años) involucradas actualmente en una relación de pareja heterosexual. De éstas, 79\% corresonde a mujeres casadas, $14 \%$ vive en unión libre y $7 \%$ se encuentra en la etapa de noviazgo o similar. La moda de escolaridad fue de licenciatura. Eran empleadas 37\%, 24\% se dedicaba al hogar y el restante $39 \%$ corresponde a profesionistas, trabajadoras independientes o empresarias. Contaban, en promedio, con 14 años de relación, con un rango de 44.5 años, y un promedio de dos hijos.

\section{Instrumentos}

Se emplearon las siguientes escalas:

- Escala de Motivación Sexual (García, 2007). Esta escala se empleó para evaluar el deseo sexual, y consiguió su validez de constructo mediante un análisis factorial con rotación ortogonal (Varimax), de donde se obtuvieron ocho factores congruentes con valor propio mayor a 1 que explicaban $60.94 \%$ de la varianza. La escala quedó conformada por un total de 67 reactivos distribuidos en ocho dimensiones. La consisten- cia interna de la escala, conseguida mediante el alfa de Cronbach fue de 0.9488 .

- Inventario Multidimensional de Infidelidad (IMIN) (Romero, Rivera y Díaz-Loving, 2007). Se usó la versión corta que consta de:

- La Subescala de Conducta Infiel: contó con los cinco reactivos de cada factor con mayor peso factorial. En total, 20 reactivos tipo Likert, con cinco opciones de respuestas que van de "nunca" a "siempre", miden la conducta infiel emocional y sexual, así como el deseo de llevar a cabo la infidelidad sexual y el deseo de llevar a efecto la infidelidad emocional.

- La Subescala de Motivos de Infidelidad. La versión corta contó con los cinco reactivos de cada factor con mayor peso factorial. En total 35 reactivos tipo Likert, con cinco opciones de respuestas que van de "totalmente de acuerdo" hasta "totalmente en desacuerdo", que miden los diferentes motivos por los cuales se podría llevar a cabo el acto de infidelidad.

- La Subescala de Concepto de Infidelidad. La versión corta contó con los cinco reactivos de cada factor con mayor peso factorial. En total 30 reactivos tipo Likert, con cinco opciones de respuesta, que van de "muchísimo" a "nada", y miden la connotación que los individuos le atribuyen a la infidelidad.

- El Inventario de Orientación Sociosexual (García, 2007). Esta escala obtuvo su validez de constructo a través de un análisis factorial con rotación ortogonal (Varimax), y se consiguieron dos factores que explican $73.37 \%$ de la varianza. Consta de siete reactivos: tres preguntas abiertas (acerca del número de parejas sexuales pasadas y esperadas) y cuatro afirmaciones que se responden con escala tipo Likert de nueve opciones de respuesta, desde "totalmente en desacuerdo" hasta "totalmente de acuerdo".

- El Inventario de Conducta Sexual (García \& Díaz Loving, 2007). Esta escala logró su validez de constructo mediante un análisis factorial con rotación ortogonal (Varimax), obteniéndo- 
se cinco factores con valores propios mayores a uno, que explican $49.55 \%$ de la varianza. La consistencia interna de la escala, obtenida a través del alfa de Cronbach fue de 0.9599 .

- La Escala de Personalidad Sexual (Díaz Loving, Rivera, Rocha, Sánchez \& Schmith, 2002). Esta escala obtuvo su validez de constructo a través de un análisis factorial con rotación ortogonal (Varimax), obteniéndose 10 factores congruentes, con valor propio mayor a 1 y que explicaban $49 \%$ de la varianza.

- La Escala de Celos (Díaz Loving, Rivera, \& Flores, 1986). Esta escala obtuvo su validez mediante un análisis factorial de componentes principales y con rotación varimax, el cual arrojo 12 factores con valor propio mayor de 1 , los cuales explicaban $69.8 \%$ de la varianza total. Asimismo, se eligió sólo aquellos reactivos que presentaran un peso factorial mayor o igual a 0.40 . Estos factores de acuerdo con su contenido conceptual, fueron divididos en dos dimensiones, los primeros seis factores se englobaron en el área denominada "Emociones y sentimientos" y los siguientes seis, en la dimensión de "Cogniciones y estilos".

- Escala de Satisfacción Marital (Cortés, Reyes, Díaz-Loving, Rivera \& Monjaraz, 1994). Esta escala obtuvo su validez mediante un análisis factorial de componentes principales, con rotación Varimax para lograr la validez de constructo del instrumento, del cual se eligieron seis factores con un valor propio mayor de 1 , los cuales explicaban $68.8 \%$ de la varianza. Asimismo, se seleccionó sólo aquellos reactivos que tenían un peso factorial mayor o igual a 0.40 para conformar el instrumento final. La versión corta consta de 48 reactivos.

\section{Procedimiento}

La aplicación de los instrumentos se llevó a cabo en lugares como: escuela, trabajo, centro de atención psicológica, calle, entre otros.

Se pidió a las participantes que respondieran de la manera más clara y sincera posible, destacando que el cuestionario formaba parte de una investigación cuyo objetivo era conocer cómo son las relaciones de pareja.
Asimismo, se destacó el anonimato de las respuestas y se les dijo que éstas no serían catalogadas como buenas o malas, correctas o incorrectas, con el fin de dar confianza a las participantes.

\section{RESULTADOS}

Con el fin de reducir las variables antes de llevar a cabo la prueba del modelo, para reducir la probabilidad de multicolinealidad entre las variables y evitar problemas al ingresar las variables a la ecuación de regresión, se llevó a cabo un análisis factorial de segundo orden para las variables propuestas en el modelo.

El análisis de componentes principales mostró nueve factores con comunalidades menores a 0.40 , por lo que fueron excluidos del análisis los factores de: restricción sexual, personalidad sexual infiel, insatisfacción con la relación primaria, satisfacción con los hijos, confianza-desconfianza, dolor y confianza.

Al realizar el análisis factorial con rotación ortogonal (Varimax), se detectaron siete factores con valores propios mayores a uno, que explicaron $73.2 \%$ de la varianza. El punto de cohorte para incluir algún factor en una dimensión fue de 0.40 y las cargas factoriales oscilaron entre 0.90 y 0.55 , lo que indica una consistencia interna suficiente. Los factores que no lograron agruparse en alguna dimensión se eliminaron.

Posteriormente, se llevaron a cabo análisis de regresión jerárquica múltiple paso por paso para detectar cuáles de estos factores serían predictores confiables del deseo sexual en mujeres.

\section{Vinculación deficiente}

El análisis de regresión constó de dos pasos en los que se detectó que los predictores más importantes fueron la personalidad sexual conquistadora y la promiscuidad. Aquellas mujeres que reportaron una personalidad sexual excitante, atractiva, conquistadora, seductora, provocativa, etc., y que reportaron estar menos dispuestas a la promiscuidad, la infidelidad, la aventura y la lujuria, presentaron una alta frecuencia de problemas en el funcionamiento y dinámica del vínculo interpersonal que inhiben la conducta sexual, tales como falta de afecto, de deseo y de interés, características denominadas vinculación deficiente. Los demás factores fueron excluidos del modelo. 
Tabla 1. Factores de segundo orden obtenidos

\begin{tabular}{|c|c|c|}
\hline FACTOR & ALFA & INDICADORES \\
\hline Celos & $a=.937$ & $\begin{array}{l}\text { Temor, frustración, actitud negativa hacia la relación, obsesión, control, } \\
\text { respuestas emocionales generadas por celos, enojo, desconfianza y } \\
\text { suspicacia-intriga. }\end{array}$ \\
\hline $\begin{array}{l}\text { Satisfacción con la } \\
\text { relación }\end{array}$ & $a=.897$ & $\begin{array}{l}\text { Satisfacción con la interacción, el atractivo físico-sexual, la diversión en } \\
\text { la pareja, la organización y el funcionamiento, así como la familia. }\end{array}$ \\
\hline Personalidad impulsiva & $a=.856$ & $\begin{array}{l}\text { Inestabilidad emocional y social, impulsividad, agresión, sexualidad } \\
\text { y apatía. }\end{array}$ \\
\hline Infidelidad & $a=.772$ & $\begin{array}{l}\text { Infidelidad emocional, infidelidad sexual, deseo de infidelidad sexual, } \\
\text { deseo de infidelidad emocional. }\end{array}$ \\
\hline $\begin{array}{l}\text { Personalidad } \\
\text { conquistadora }\end{array}$ & $a=.892$ & $\begin{array}{l}\text { Personalidad atractiva involucrada, atractiva sexual, conquistadora } \\
\text { y liberal. }\end{array}$ \\
\hline Inseguridad & $a=.742$ & $\begin{array}{l}\text { Inseguridad, temor al abandono por insatisfacción y sentimiento } \\
\text { de pérdida. }\end{array}$ \\
\hline Promiscuidad & $a=.730$ & Promiscuidad, masculinidad, lujuria, personalidad sexual perversa. \\
\hline
\end{tabular}

Tabla 2. Predictores confiables para la vinculación deficiente

\begin{tabular}{c|c|c|c|c|c|c|c|c}
\hline & Modelo & B & Sig. & $\begin{array}{c}\text { Cambio de } \\
\text { la F }\end{array}$ & $\begin{array}{c}\text { Sig. del cambio } \\
\text { de la F }\end{array}$ & $\begin{array}{c}\mathbf{R} \\
\text { múltiple }\end{array}$ & $\begin{array}{c}\text { Error } \\
\text { estándar }\end{array}$ \\
\hline 2 & $\begin{array}{c}\text { Personalidad } \\
\text { conquistadora }\end{array}$ & 0.233 & 0.000 & 12.936 & 0.000 & 0.354 & 0.126 & 1.000 \\
\hline & Promiscuidad & -0.307 & 0.001 & & & & \\
\hline
\end{tabular}

Tabla 3. Predictores confiables para la falta de deseo sexual

\begin{tabular}{l|c|c|c|c|c|c|c|c}
\hline & Modelo & B & Sig. & $\begin{array}{c}\text { Cambio } \\
\text { de la F }\end{array}$ & $\begin{array}{c}\text { Sig. del cambio } \\
\text { de la F }\end{array}$ & $\begin{array}{c}\mathbf{R} \\
\text { múltiple }\end{array}$ & $\begin{array}{c}\text { Error } \\
\text { estándar }\end{array}$ \\
\hline 3 & $\begin{array}{c}\text { Personalidad } \\
\text { impulsiva }\end{array}$ & 0.279 & 0.002 & 9.626 & 0.000 & 0.373 & 0.139 & 0.938 \\
\hline & $\begin{array}{c}\text { Personalidad } \\
\text { conquistadora }\end{array}$ & 0.175 & 0.002 & & & & & \\
\hline & Promiscuidad & -0.195 & 0.018 & & & & \\
\hline
\end{tabular}




\section{Falta de deseo sexual}

En el caso de la falta de deseo sexual, el análisis de regresión múltiple constó de tres pasos. Se detectó que los predictores más importantes fueron la personalidad impulsiva, la personalidad sexual conquistadora y la promiscuidad. Las mujeres de la muestra que presentaban una personalidad impulsiva (inestable, impulsiva, agresiva y apática), una personalidad sexual conquistadora y poca promiscuidad contaban con falta de deseo sexual, que se refiere a la ausencia del impulso o la energía sexual necesarios para que haya actividad sexual (vid. tabla 3). Los demás factores fueron excluidos del modelo.

\section{Expresión afectiva}

El análisis de regresión múltiple para la expresión afectiva constó de dos pasos. Se detectó que los predictores más importantes fueron la personalidad sexual conquistadora y la promiscuidad. Aquellas mujeres que reportaron una personalidad sexual excitante, atractiva, conquistadora, seductora, provocativa, etc., y que reportaron estar menos dispuestas a la promiscuidad (a la infidelidad, la aventura y la lujuria) presentaron una alta frecuencia en expresión afectiva. Los demás factores fueron excluidos del modelo ( $v i d$. tabla 4 ).

\section{Atracción interpersonal}

El análisis de regresión constó de tres pasos en los que se detectó que los predictores más importantes fueron la personalidad sexual conquistadora, la promiscuidad y la personalidad impulsiva. Las mujeres de la muestra que presentaban una personalidad sexual conquistadora, poca promiscuidad y una baja personalidad impulsiva (inestable, impulsiva, agresiva y apática) contaban con altos puntajes en atracción interpersonal, que se refiere a características de evaluación del otro(a) que favorecen la actividad sexual (vid. tabla 5). Los demás factores fueron excluidos del modelo.

\section{Placer físico}

En el caso de la personalidad sexual abierta, el análisis de regresión múltiple constó de tres pasos. Se detectó que los predictores más importantes fueron nuevamente la personalidad sexual conquistadora, la promiscuidad

Tabla 4. Predictores confiables para la personalidad sexual restrictiva

\begin{tabular}{c|c|c|c|c|c|c|c|c}
\hline & Modelo & B & Sig. & $\begin{array}{c}\text { Cambio } \\
\text { de la F }\end{array}$ & $\begin{array}{c}\text { Sig. delcambio } \\
\text { de la F }\end{array}$ & $\begin{array}{c}\mathbf{R} \\
\text { múltiple }\end{array}$ & $\begin{array}{c}\text { Error } \\
\text { estándar }\end{array}$ \\
\hline 2 & $\begin{array}{c}\text { Personalidad } \\
\text { conquistadora }\end{array}$ & 0.304 & 0.000 & 25.420 & 0.000 & 0.469 & 0.220 & 0.789 \\
\hline & Promiscuidad & -0.201 & 0.004 & & & & & \\
\hline
\end{tabular}

Tabla 5. Predictores confiables para la atracción interpersonal

\begin{tabular}{c|c|c|c|c|c|c|c|c}
\hline & Modelo & B & Sig. & $\begin{array}{c}\text { Cambio } \\
\text { de la F }\end{array}$ & $\begin{array}{c}\text { Sig. del cambio } \\
\text { de la F }\end{array}$ & $\begin{array}{c}\mathbf{R} \\
\text { múltiple }\end{array}$ & $\begin{array}{c}\text { Error } \\
\text { estándar }\end{array}$ \\
\hline \multirow{3}{*}{3} & $\begin{array}{c}\text { Personalidad } \\
\text { conquistadora }\end{array}$ & 0.375 & 0.000 & 19.214 & 0.000 & 0.494 & 0.224 & 0.905 \\
\hline & $\begin{array}{c}\text { Promiscuidad } \\
\end{array}$ & -0.262 & 0.001 & & & & & \\
\hline
\end{tabular}


y la inseguridad. Las mujeres de la muestra que presentaban una personalidad sexual conquistadora contaban con baja promiscuidad y sentían temor por perder a su pareja, mostraban una mayor búsqueda de la consumación erótica, dar o experimentar satisfacción física a través de las sensaciones corporales placenteras, que actúan como estímulos que favorecen la conducta sexual (vid. tabla 6).

\section{Deseo sexual}

En el caso del deseo sexual, el análisis de regresión múltiple constó de dos pasos. Se detectó que los predictores más importantes fueron, nuevamente, la personalidad sexual conquistadora y la promiscuidad. Las participantes con una personalidad sexual conquistadora y baja promiscuidad tenderán con mayor probabilidad a requerir un impulso o energía sexual ya sea personal o del compañero(a) para la actividad sexual (vid. tabla 7).

\section{Obstáculos}

El análisis de regresión constó de cuatro pasos en los que se detectó que los predictores más importantes de los obstáculos en la motivación sexual fueron la personalidad impulsiva, la satisfacción con la relación, los celos y la promiscuidad. Aquellas participantes que reportaron elementos personales y situacionales que dificultan la conducta sexual (obstáculos), presentaron una alta personalidad impulsiva y celos hacia su pareja, así como una baja satisfacción con la relación y bajos niveles de promiscuidad (vid. tabla 8 ).

Tabla 6. Predictores confiables para el placer físico

\begin{tabular}{c|c|c|c|c|c|c|c|c}
\hline & Modelo & B & Sig. & $\begin{array}{c}\text { Cambio } \\
\text { de la F }\end{array}$ & $\begin{array}{c}\text { Sig. del cambio } \\
\text { de la F }\end{array}$ & $\begin{array}{c}\text { R } \\
\text { múltiple }\end{array}$ & $\mathbf{R}^{2}$ & $\begin{array}{c}\text { Error } \\
\text { estándar }\end{array}$ \\
\hline 3 & $\begin{array}{c}\text { Personalidad } \\
\text { conquistadora }\end{array}$ & 0.426 & 0.000 & 33.772 & 0.000 & 0.601 & 0.361 & 0.776 \\
\hline & Promiscuidad & -0.249 & 0.000 & & & & & \\
\hline & Inseguridad & 0.133 & 0.021 & & & & \\
\hline
\end{tabular}

Tabla 7. Predictores confiables para el deseo sexual

\begin{tabular}{l|c|c|c|c|c|c|c|c}
\hline & Modelo & B & Sig. & $\begin{array}{c}\text { Cambio } \\
\text { de la F }\end{array}$ & $\begin{array}{c}\text { Sig. del cambio } \\
\text { de la F }\end{array}$ & $\begin{array}{c}\text { R } \\
\text { múltiple }\end{array}$ & $\begin{array}{c}\text { R } \\
\text { estándar }\end{array}$ \\
\hline 2 & $\begin{array}{c}\text { Personalidad } \\
\text { conquistadora }\end{array}$ & 0.365 & 0.000 & 30.649 & 0.000 & 0.504 & 0.254 & 0.918 \\
\hline & Promiscuidad & -0.348 & 0.000 & & & & & \\
\hline
\end{tabular}

Tabla 8. Predictores confiables para obstáculos

\begin{tabular}{c|c|c|c|c|c|c|c|c}
\hline & Modelo & B & Sig. & $\begin{array}{c}\text { Cambio } \\
\text { de la F }\end{array}$ & $\begin{array}{c}\text { Sig. del cambio } \\
\text { de la F }\end{array}$ & $\begin{array}{c}\text { R } \\
\text { múltiple }\end{array}$ & $\begin{array}{c}\text { Error } \\
\text { estándar }\end{array}$ \\
\hline 4 & Personalidad impulsiva & 0.426 & 0.000 & 14.876 & 0.000 & 0.501 & 0.251 & 0.823 \\
\hline & Satisfacción & -0.325 & 0.000 & & & & \\
\hline & Celos & 0.277 & 0.002 & & & & & \\
\hline & Promiscuidad & -0.175 & 0.015 & & & & \\
\hline
\end{tabular}




\section{Facilitadores}

Finalmente, en el caso de la personalidad de los facilitadores de la motivación sexual, el análisis de regresión múltiple constó de dos pasos. Se detectó que los predictores más importantes, una vez más, fueron la personalidad sexual conquistadora y la promiscuidad. Aquellas mujeres que reportaron una personalidad sexual excitante, atractiva, conquistadora, seductora, provocativa, etc., y que reportaron estar menos dispuestas a la promiscuidad, la infidelidad, la aventura y la lujuria reportaron elementos del ambiente o contexto físico y social que favorecen la conducta sexual, como lo adecuado del ambiente y de la ocasión y la disponibilidad de un compañero (a) sexual. Los demás factores fueron excluidos del modelo (vid. tabla 9).

\section{DISCUSIÓN}

A partir de los resultados que se obtuvieron en la presente investigación, es posible inferir que el desarrollo de una personalidad sexual estará íntimamente relacionado con la motivación y el deseo sexual (Buss, 2005).

Como se puede observar, la motivación sexual tuvo como principales predictores una personalidad sexual conquistadora y la baja tendencia al factor denominado promiscuidad. Esto indica que las mujeres de esta muestra presentarán una actitud conquistadora cuando tengan una alta motivación o deseo sexual. Una mujer típica de la muestra se comportará de manera conquistadora, abierta, atractiva, sensual y seductora cuando deseé experimentar el enamoramiento hasta llegar a la conducta sexual.

Resulta de suma importancia destacar que, de acuerdo con los modelos obtenidos, el deseo o motivación sexual se presenta más en mujeres cuando és- tas tienden menos a la promiscuidad, es decir, cuando reportan una actitud negativa hacia la infidelidad, la aventura, la lujuria, el sadomasoquismo, etc. Este hallazgo puede deberse a que en culturas tradicionales como la mexicana, la normatividad del deseo sexual sigue siendo distinta para hombres y mujeres. Es mucho más restrictiva para ellas aun en el medio urbano actual, donde esa restricción se encuentra tan asimilada que ellas mismas funcionan como su propio actor de restricción. Todavía en la actualidad, y a pesar de que las mujeres han ganado espacios en múltiples áreas -como la laboral, social, política y pública (Rocha \& Cruz del Castillo, 2013)-, este empoderamiento parece no ser tan flexible en la sexualidad, donde el estándar romántico conquistador sigue siendo el predominante. En el área privada se sigue autocastigando el deseo abierto de la sexualidad y ésta se vincula exclusivamente al ámbito del enamoramiento, enmarcado en una relación de pareja estable.

Cabe resaltar el hecho de que las características de personalidad asociadas a la impulsividad, la inestabilidad social y emocional, así como la ideología tradicional hayan tomado relevancia en el estudio, en principio al constituirse en un factor de segundo orden, y después al resultar en predictores importantes de la falta de deseo sexual, la atracción interpersonal y los obstáculos de la motivación sexual. Esto corrobora -como lo menciona la literatura previa- la influencia de características neuróticas en el desarrollo de la personalidad sexual (Eysenck, 1976; Miller, Lynam, Zimmerman, Logan, Leukefeld \& Clayton, 2000; Wright \& Reise, 1997).

El hecho de autopercibirse como una persona inestable e impulsiva puede orientar a una mujer hacia la ausencia del impulso o la energía sexual, necesarios para

Tabla 9. Predictores confiables para facilitadores

\begin{tabular}{c|c|c|c|c|c|c|c|c}
\hline & Modelo & B & Sig. & $\begin{array}{c}\text { Cambio } \\
\text { de la F }\end{array}$ & $\begin{array}{c}\text { Sig. del cambio } \\
\text { de la F }\end{array}$ & $\begin{array}{c}\mathbf{R} \\
\text { múltiple }\end{array}$ & $\begin{array}{c}\text { Error } \\
\text { estándar }\end{array}$ \\
\hline 2 & $\begin{array}{c}\text { Personalidad } \\
\text { conquistadora }\end{array}$ & 0.276 & 0.000 & 19.401 & 0.000 & 0.421 & 0.177 & 0.868 \\
\hline & Promiscuidad & -0.257 & 0.001 & & & & & \\
\hline
\end{tabular}


que haya actividad sexual; hacia una menor atracción interpersonal con la pareja sexual y hacia el desarrollo de elementos personales y situacionales que dificulten la conducta sexual, como la tristeza, la inseguridad, las presiones y la falta de un compañero(a) sexual.

Estos hallazgos indicarían que el desarrollo de la personalidad sexual se encuentra íntimamente ligado con los objetivos sexuales que persigan las mujeres; hallazgo que apoya algunas teorías evolutivas, como la teoría de la inversión parental (Buunk \& Dijkstra, 2000; Symons, 1979; Trivers 1972), y la teoría de las estrategias sexuales (Barash \& Lipton, 2002; Buss \& Schmitt, 1993).

\section{REFERENCIAS}

Barash, D. P. \& Lipton, J. E. (2002). The myth of monogamy: Fidelity and infidelity in animals and people. New York: Holt Paperbacks.

Basson, R. (2001). Using a different model of female sexual response to address women's problematic low sexual desire. Journal of Sex \& Marital Therapy, 27, 395-403.

Buss, D. M. \& Schmitt, D. P. (1993). Sexual strategies theory: an evolutionary perspective on human mating. Psychological Review, 100, 204-232.

Buss, D. M. (2005). The Handbook of Evolutionary Psychology. Nueva York: John Wiley \& Sons.

Buunk, B. P. \& Dijkstra, P. (2000). Extradyadic Relationships and Jealously. En C. Hendrick \& S. Hendrick (Eds.) (2000). Close Relationships. A Sourcebook. California: Sage.

Cortés, M. S., Reyes, D., Díaz Loving, R., Rivera, A. S. \& Monjaraz, C. J. (1994). Elaboración y análisis psicométrico del Inventario Multifacético de Satisfacción Marital. La Psicología Social en México. Asociación Mexicana de Psicología Social 5.

Cruz del Castillo, C., Romero, A. \& Gil-Bernal, F. M. E. (2013). Indicadores de deseo, autoerotismo e impulsividad sexual en mujeres de la Ciudad de México. Acta de investigación psicológica, 3(1), 1031-1040.

Dennerstein, L., Hayes, R., Sand, M. \& Lehert, P. (2009). Attitudes toward and frequency of partner interactions among women reporting decreased sexual desire. Journal of Sexual Medicine, 6, 1668-1673.
El campo tanto de la motivación como de la personalidad sexual ha sido poco explorado aún, principalmente en México. La investigación y los resultados al respecto son escasos. De ahí la relevancia de los resultados empíricos asociados a la personalidad sexual. Es indispensable, por tanto, continuar con el desarrollo de los estudios que vinculen la personalidad sexual con otras variables y fenómenos de las interacciones sociales, capaces de crear modelos tanto de predicción como de explicación de la personalidad sexual. •

Díaz-Loving, R., Rivera, A. S. \& Flores, G. M. (1986). Celos: reacciones ante la posible pérdida de la pareja. La Psicología Social en México, 1, 386-391.

Díaz-Loving, R., Rivera, A. S., Rocha, S. T., Sánchez, A. R. \& Schmith, D. (2002). Marcado por la conquista: rasgos de personalidad derivados de la vida sexual. Revista de Psicología Social y Personalidad, 18(1), 77-92.

Eysenck, H. J. (1976). Sex and personality. London: Open Books.

García, G. (2007). Conducta sexual: el papel de la biología, la psicología y la sociocultura. Tesis de Doctorado. Facultad de Psicología. Universidad Nacional Autónoma de Mexico.

García, G. \& Díaz-Loving, R. (2007) Expresiones afectivas y sexuales en diversos contextos relacionales. En Crisis, expresiones sexuales y celos en las relaciones de pareja. Simposio efectuado en el xxxi Congreso Interamericano de Psicología, Distrito Federal, México.

Giles, J. (2006). Social constructionism and sexual desire. Journal for the Theory of Social Behaviour, 36(3), 225-237.

Kamei, 1. \& Kamei, J. (2005). Estudio de prevalencia de los trastornos de la sexualidad en mujeres que asisten a consultorio externo de gineco-obstetricia del hospital La Serena. Revista Chilena de Urología, 70(4), 231-235.

Kingsberg, S. (2011). Trastorno de deseo sexual hipoactivo: comprensión del efecto en las mujeres de mediana edad. Revista del climaterio, 14(83), 175-178. 
Levine, S. B. (1992). The paradoxes of Sexual Desire. En Sexual life: A clinician's guide. Plenum Press: Nueva York.

Levine, S. B. (2003). The Nature of Sexual Desire: A Clinician's Perspective. Archives of Sexual Behavior, 32, 3.

Miller, J. D., Lynam, D. R., Zimmerman, R., Logan, T., Leukefeld, C. \& Clayton, R. (2000). Risky sexual behaviour and the five factor model of personality. En Meeting of the American Psychological Association. Washington, D.C.: American Psychological Association.

Regan, P.C. \& Berscheid, E. (2000). Lust: What we know about human sexual desire. Thousands Oaks, California: Sage.

Rocha, T. \& Cruz del Castillo, C. (2013). Mujeres en transición. Reflexiones teórico-empíricas en torno a la sexualidad, la pareja y el género. México: Universidad Iberoamericana.

Romero, A., Cruz, C., \& Díaz Loving, R., (2008). Propuesta de un modelo Bio-Psico-Socio-Cultural de Infidelidad Sexual y Emocional en Hombres y Mujeres. Psicología Iberoamericana, 16(2), 14-21.
Romero, A., Rivera, S. \& Díaz Loving, R. (2007). Desarrollo del Inventario Multidimensional de Infidelidad (IMIN). Revista Iberoamericana de Diagnóstico y Evaluación en Psicología, 23(1), 121-148.

Sánchez, C., Corres, N., Blum, B. \& Carreño, J. (2009). Perfil de la relación de factores psicológicos del deseo sexual hipoactivo femenino y masculino. Salud Mental, 32, 43-51.

Shifren, J. L., Monz, B. U., Russo, P. A., Segreti, A. \& Johannes, C. B. (2008). Sexual problems and distress in United States women: prevalence and correlates. Obstetrics \& Gynecology, 112(5), 970-978.

Symons, D. (1979). The evolution of human sexuality. Nueva York: Oxford.

Trivers, R. (1972). Parental investment and sexual selection. En B. Campbell (Ed). Sexual selection and the descent of man, 1871-1971 (pp. 136-179). Chicago: Adline.

Wright, T. M. \& Reise, S. P. (1997). Personality and unrestricted sexual behavior: correlations of sociosexuality in caucasian and Asian College students. Journal of Research in Personality, 31, 166-192. 